ARTICLE

\title{
Control of cotton fibre elongation by a homeodomain transcription factor GhHOX3
}

Chun-Min Shan ${ }^{1}$, Xiao-Xia Shangguan ${ }^{1}$, Bo Zhao ${ }^{1}$, Xiu-Fang Zhang ${ }^{1}$, Lu-men Chao ${ }^{1}$, Chang-Qing Yang ${ }^{1}$, Ling-Jian Wang ${ }^{1}$, Hua-Yu Zhu², Yan-Da Zeng², Wang-Zhen Guo², Bao-Liang Zhou², Guan-Jing Hu ${ }^{3}$, Xue-Ying Guan ${ }^{2,4}$, Z Jeffrey Chen ${ }^{2,4}$, Jonathan F. Wendel ${ }^{3}$, Tian-Zhen Zhang ${ }^{2} \&$ Xiao-Ya Chen ${ }^{1,5}$

Cotton fibres are unusually long, single-celled epidermal seed trichomes and a model for plant cell growth, but little is known about the regulation of fibre cell elongation. Here we report that a homeodomain-leucine zipper (HD-ZIP) transcription factor, GhHOX3, controls cotton fibre elongation. GhHOX3 genes are localized to the 12th homoeologous chromosome set of allotetraploid cotton cultivars, associated with quantitative trait loci (QTLs) for fibre length. Silencing of GhHOX3 greatly reduces (>80\%) fibre length, whereas its overexpression leads to longer fibre. Combined transcriptomic and biochemical analyses identify target genes of GhHOX3 that also contain the L1-box cis-element, including two cell wall loosening protein genes GhRDL1 and GhEXPA1. GhHOX3 interacts with GhHD1, another homeodomain protein, resulting in enhanced transcriptional activity, and with cotton DELLA, GhSLR1, repressor of the growth hormone gibberellin (GA). GhSLR1 interferes with the GhHOX3-GhHD1 interaction and represses target gene transcription. Our results uncover a novel mechanism whereby a homeodomain protein transduces GA signal to promote fibre cell elongation.

\footnotetext{
${ }^{1}$ National Key Laboratory of Plant Molecular Genetics, National Plant Gene Research Center, Institute of Plant Physiology and Ecology, Shanghai Institutes for Biological Sciences, Chinese Academy of Sciences, Shanghai 200032, China. ${ }^{2}$ National Key Laboratory of Crop Genetics \& Germplasm Enhancement, MOE Hybrid Cotton R\&D Engineering Research Center, Nanjing Agricultural University, Nanjing 210095, China. ${ }^{3}$ Department of Ecology, Evolution and Organismal Biology, lowa State University, Ames, lowa 50011, USA. ${ }^{4}$ Department of Molecular Biosciences, Center for Computational Biology and Bioinformatics, and Institute for Cellular and Molecular Biology, The University of Texas at Austin, Austin, Texas 78712, USA. ${ }^{5}$ Plant Science Research Center, Shanghai Chenshan Botanical Garden, Shanghai 201602, China. Correspondence and requests for materials should be addressed to X.-Y.C. (email: xychen@sibs.ac.cn) or to T.-Z.Z. (email: cotton@njau.edu.cn).
} 
C otton is widely grown in over 80 countries with an annual output value of $\$ 12$ billion, mainly for cotton fibre, the most important natural and renewable material for the textile industry ${ }^{1}$. Fibre length is a key trait determining cotton quality and yield. In addition, the extensively elongated fibre cells, which undergo semi-synchronized development and rapid growth, provide an excellent system for the study of cell elongation ${ }^{2}$. Fibre development can be divided into four overlapping stages: initiation, elongation, secondary cell wall biosynthesis and maturation ${ }^{3}$. Although progress has been made in the identification of regulators controlling cotton fibre initiation (GhMYB25 and GhMYB25-like) ${ }^{4,5}$, and a number of factors have been proposed to affect fibre cell growth ${ }^{6-11}$, to date the key regulators of fibre elongation have not been identified, nor has the regulatory mechanism been elucidated.

Here we show that a homeodomain-leucine zipper (HD-ZIP) transcription factor, GhHOX3, plays a central role in controlling cotton fibre elongation. We also elucidate a molecular link between the phytohormone gibberellin (GA) and the homeobox regulators in promoting plant cell growth.

\section{Results}

GhHOX3 associates with cotton fibre traits. The two widely cultivated cotton species, Gossypium hirsutum $(\mathrm{AD})_{1}$ and G. barbadense $(\mathrm{AD})_{2}$, are allotetraploids containing $\mathrm{A}$ - and D-homoeologous genomes, and among the diploids only the A-genome species produce spinnable fibre ${ }^{12}$. In Arabidopsis thaliana, the HD-ZIP IV subfamily factor GLABRA2 (AtGL2) is a positive regulator of the trichome development, functioning downstream of the initiation complex ${ }^{13}$. We previously reported that three HD-ZIP IV factors of cotton, HOX1/2/3 (Supplementary Fig. 1a,b), are expressed in cotton fibre cells ${ }^{14,15}$. Southern blots showed double copies of GhHOX3 in G. hirsutum and a single copy in the two models of the extant progenitor diploids, $G$. herbaceum $\left(\mathrm{A}_{1}\right)$ and $G$. raimondii $\left(\mathrm{D}_{5}\right.$; Supplementary Fig. 2), consistent with the sequenced $G$. raimondii genome ${ }^{12}$. The homoeologous GhHOX3-A and GhHOX3-D share 98\% aminoacid sequence identity (Supplementary Fig. 1b). On the basis of single-nucleotide polymorphisms (SNPs) in the the A- and D-homoeologous sequences between G. hirsutum acc. TM-1 and G. barbadense cv. Hai7124 (ref. 16), GhHOX3 genes were mapped on the 12th homoeologous chromosome pairs, A12 (Chr. 12) and D12 (Chr. 26; Supplementary Fig. 3), whereas GhHOX1 and GhHOX2 were localized to the fifth and the third homoeologous sets, respectively. By integrating with previously anchored quantitative trait loci within $20 \mathrm{cM}$, both $G h H O X 3-A$ and GhHOX3-D were associated with the quantitative trait loci for fibre length, uniformity and fineness (Supplementary Table 1). We subsequently surveyed 281 Chinese cultivars for SNP variation in GhHOX3-A (A12) and detected two SNPs, at positions 2560 $(\mathrm{C} / \mathrm{T})$ and $2761(\mathrm{G} / \mathrm{A})$. Association analysis showed that these two SNPs were significantly correlated with fibre length and uniformity in nine environments (Supplementary Table 2).

GhHOX3 regulates cotton fibre elongation. Designed to overexpress GhHOX genes in cotton, we used the $35 S$ promoter to drive the cDNA and transferred the constructs into G. hirsutum. While the 35S::GhHOX1 and 35S::GhHOX2 plants did not exhibit clear phenotypic effects, the 35S::GhHOX3 plants showed a dramatic decrease of fibre length (Supplementary Table 3), which was more pronounced in homozygous lines of $\mathrm{T}_{2}$ (Fig. 1a) and subsequent generations ( $>80 \%$ decrease). Cotton cultivars produce two types of fibre: the longer spinnable 'lint', and the shorter non-spinnable 'fuzz'. Fibres of the homozygous transgenic lines
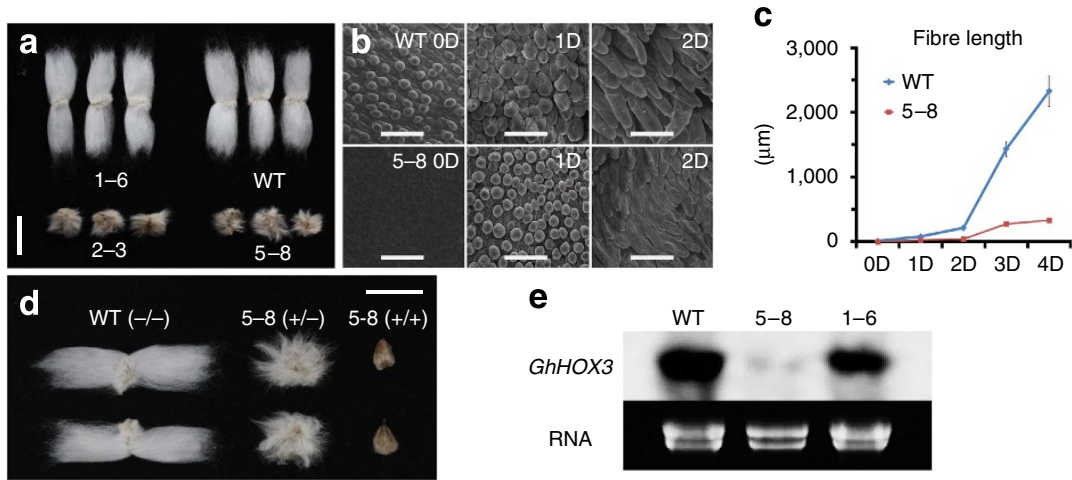

$\mathbf{e}$
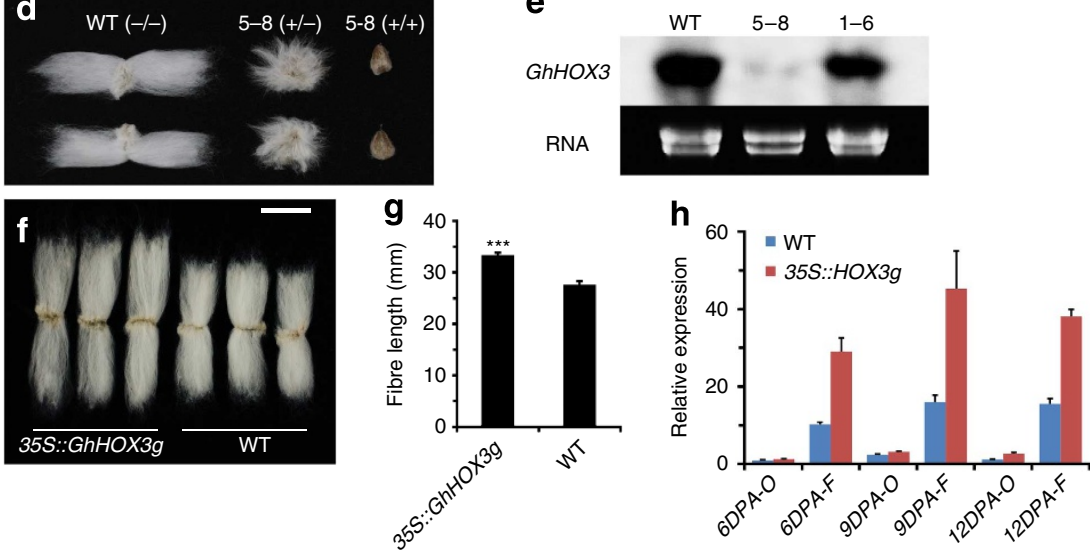

Figure 1 | GhHOX3 expression affects cotton fibre elongation. (a-e) Phenotypic analysis of 35S::GhHOX3 transgenic plants. Wild-type (WT) cotton and three transgenic lines are shown, of which two (5-8 and 2-3) exhibited co-suppression. (a) Images of fibre of 35S::GhHOX3 lines, $T_{2}$ generation. Scale bar, $1 \mathrm{~cm}$. (b) Scanning electron microscope (SEM) images of the ovule (0-2 DPA) of the co-suppression line 5-8 and the WT. Scale bars, $50 \mu \mathrm{m}$. (c) Mean fibre length at 0-4 DPA (mean \pm s.e.m., $n>100$ ). (d) Fibre of homozygous and heterozygous 35S::GhHOX3 co-suppression line 5-8 ( $T_{6}$ generation)

Heterozygotes were generated by back-crossing the $T_{5}$ plants to the WT. Scale bar, $1 \mathrm{~cm}$. (e) Northern blot of GhHOX3 expression in fibre (9 DPA) of the WT and the 35S::GhHOX3 (5-8 and 1-6) lines. (f) Images of fibre from a $\mathrm{T}_{2}$ plant of the 35S::GhHOX3g (genomic) transgenic line. Scale bar, $1 \mathrm{~cm}$. (g) Mature fibre length of a 355 :: GhHOX3g cotton line (mean \pm s.e.m., $n=30$, ${ }^{\star \star \star} P<0.001$, Student's $t$-test). (h) Quantitative reverse transcription-PCR analysis of GhHOX3 expression levels in 35S::GhHOX3g ovule (O) and fibre (F), $\mathrm{T}_{2}$; DPA or D, DPA. Data are shown as mean \pm s.e.m. $(n=3)$. 
were so short that the lint completely disappeared. Observation of $0-3$ days post anthesis (DPA) ovules from a $\mathrm{T}_{2}$ line (5-8) revealed that fibre elongation was severely retarded, whereas initiation (density) was less affected (Fig. 1b,c). Other aspects of the transgenic plants appeared normal, except for the impaired trichome development on stem and leaf veins (Supplementary Fig. 4a,b). Genetic tests showed that the transgene exhibited a semi-dominant effect in the presence of the wild-type allele (Fig. 1d). The GhHOX3 transcript level was sharply decreased in 35S::GhHOX3 transgenics (line 2-3 and 5-8), suggesting transgene co-suppression ${ }^{17}$. However, the expression levels of other HOX genes tested, such as GhHOX1 and GhHOX2, were not affected (Fig. 1e; Supplementary Fig. 5a-c).

To confirm the co-suppression results, we downregulated the three $H O X$ genes, respectively, by RNA interference. Again, only the GhHOX3 RNA interference cotton plants produced shortened fibre, and the phenotype was indistinguishable from that of co-suppression (Supplementary Fig. 6a-e). To overcome co-suppression by cDNA, we used a genomic fragment to overexpress GhHOX3 (GhHOX3-A) in cotton. Strikingly, compared with the wild-type, fibre length was increased up to $20 \%$ in the 35S::GhHOX3g lines in which GhHOX3 expression was elevated (Fig. 1f-h).

The cotton HOX3 was expressed preferentially in rapidly elongating fibre at 3-9 DPA of both allotretraploid (G. hirsutum) and diploid ( $G$. arboreum) cultivars grown in greenhouse (Supplementary Fig. 7a-c). Transcripts of both copies, namely GhHOX3-A and GhHOX3-D, were detectable in the fibre cells of G. hirsutum. When GhHOX3-A/D were fused with fluorescent proteins and transiently expressed in leaves, the signal was localized to the nucleus, consistent with their role as transcription factors (Supplementary Fig. 8).

Two cell wall proteins are direct targets of GhHOX3. To identify the genes regulated by GhHOX3, digital gene expression tag profiling was performed to compare the transcripts of developing cotton fibre cells of the wild-type and the co-suppression lines, which identified over 300 differentially expressed genes, of which 78 were downregulated in co-suppression lines compared with the wild-type, under a strict screening criteria (Supplementary Data 1 and 2). Among them, the majority were classified into biological processes of cell growth, including cell wall formation, transcriptional regulation, signal transduction and metabolism. We then analysed the expression of these 78 genes in developing fibre cells by quantitative reverse transcription-PCR, which confirmed the downregulation of 76 in the co-suppression and upregulation of 49 out of the 76 in the overexpression lines (Supplementary Fig. 9). In Arabidopsis, the HD-ZIP IV factors bind to the conserved cis-element, L1-box, of target genes ${ }^{18,19}$. Of the 49 genes selected, 18 contain at least one L1-box in their promoters (Supplementary Table 4), indicating that they could serve as targets of GhHOX3.

Putative targets of GhHOX3 included two cell wall protein genes, GhRDL1 and GhEXPA1. GhRDL1 has been shown to interact with GhEXPA1, an $\alpha$-expansin that functions in wall loosening and cell expansion ${ }^{9}$. We previously showed that the GhRDL1 promoter contains an L1-box and could be activated by cotton HOX3 in transgenic Arabidopsis ${ }^{14}$. Interestingly, the GhEXPA1 promoter also contains an L1-box. We found that the expression levels of both GhRDL1 and GhEXPA1 followed the change of GhHOX3 expression: significantly downregulated in GhHOX3-silenced but upregulated in overexpressed lines (Fig. 2a-d). Electrophoretic mobility shift assays (EMSA) showed that GhHOX3 bound to the intact but not the mutated L1-box, from both promoters (Fig. 2e-g). This result was confirmed by yeast one-hybrid assay (Fig. $2 \mathrm{~h}$ ).
Regulation of fibre elongation by GA involves GhHOX3. Phytohormones, including auxin, GA and ethylene, are important regulators of cotton fibre development ${ }^{10,20,21}$. As auxin and GAs are commonly involved in cell differentiation and growth, we tested if GhHOX3 functions downstream of a hormone. We cultured the cotton ovules (2 DPA) in vitro for 6 days, and found that $\mathrm{GA}_{3}$ promoted fibre elongation of the wild-type ovules in a dose-dependent manner, but not the GhHOX3 co-suppression ovules (Supplementary Fig. 10). Indeed, $\mathrm{GA}_{3}$ treatments significantly upregulated GhHOX3 and the downstream genes GhRDL1 and GhEXPA1 in the wild-type ovules, but their expression levels remained low in the co-suppression ovules (Fig. 2i-1). Increasing concentrations of auxin, however, did not exert an obvious effect on fibre elongation in our culture conditions, nor effect on the expression of GhRDL1, GhEXPA1 and GhHOX3 regardless of the cotton lines cultured (Supplementary Fig. 11a-d). Thus, GA plays an important role in regulating fibre elongation and GhHOX3 is required for this regulation.

To further address how GhHOX3 functions, we screened a cotton fibre cDNA library for its interacting proteins. Two-thirds of the proteins identified were putative transcription factors potentially involved in phytohormone function and plant development (Supplementary Table 5). These included GhHD1, another HD-ZIP IV subfamily protein, which was reported to have a role in trichome and only a mild effect on fibre cell development ${ }^{22}$, and GhSLR1, a DELLA protein of the GA signalling component ${ }^{23}$. The expression of GhHD1, like that of GhHOX1 and GhHOX2, was not affected in GhHOX3 co-suppression lines (Supplementary Fig. 12), suggesting again that the gene silencing was specific to GhHOX3 and the suppressed GhHOX3 expression was responsible for the shorter fibre phenotype observed. In further yeast two-hybrid assays, GhHOX3 strongly interacted with GhHD1 (Fig. 3a), and the interaction was also observed in the biomolecular fluorescence complementation $(\mathrm{BiFc}$ ) analysis (Fig. $3 \mathrm{~b}$ ) and the coimmunoprecipitation (CoIP) assay (Fig. 3c, Supplementary Fig. 17). DELLA proteins, which lack a DNA-binding domain, negatively regulate GA signalling by repressing activities of transcription factors through protein-protein interaction ${ }^{24}$. GhSLR1 is a functional orthologue of the Arabidopsis DELLA AtGAI, and widely expressed in various organs of cotton ${ }^{25}$ (Supplementary Fig. 13). The interaction between GhHOX3 and GhSLR1 was confirmed by yeast two-hybrid (Fig. 3d), BiFc (Fig. 3e) and CoIP assays (Fig. 3f, Supplementary Fig. 17). Interestingly, GhHD1 did not bind to GhSLR1 in yeast (Fig. 3d). We then tested if the cotton DELLA would compete with GhHD1 for binding to GhHOX3. Indeed, in the yeast three-hybrid assay, the GhHOX3-GhHD1 interaction was gradually weakened by increasing concentrations of GhSLR1 (Fig. 3g). Domain deletions revealed that both GhHD1 and GhSLR1 interacted with the GhHOX3 fragment containing both Leu-zipper and START domains, whereas the homeodomain was dispensable (Fig. 3h).

Given the fact that GA promotes cotton fibre elongation (Supplementary Fig. 10), we asked if the triple protein interactions would affect the transcriptional activation of target genes. A dual-luciferase assay system was employed for this purpose. The level of the luciferase activity controlled by GhRDL1 and GhEXPA1 promoters was elevated when GhHOX3 was expressed (Fig. 4a), but this activation was impaired when the L1box was mutated (Fig. 4b). GhHD1 also activated the two promoters, but to a lesser extent than GhHOX3 (Fig. 4a). The GhRDL1 L1-box conferred a higher degree of regulation, possibly due to its closer proximity to the coding region (Supplementary Table 4). Activation of GhHOX3 to GhRDL1 or GhEXPA1 promoters was significantly enhanced by GhHD1 (Fig. 4a), and 

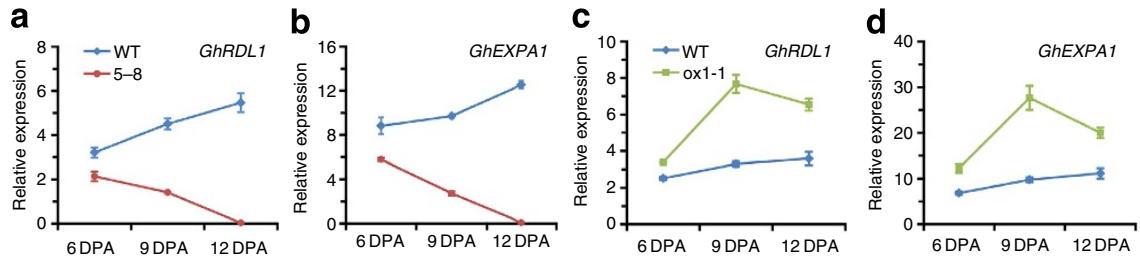

$\mathbf{f}$
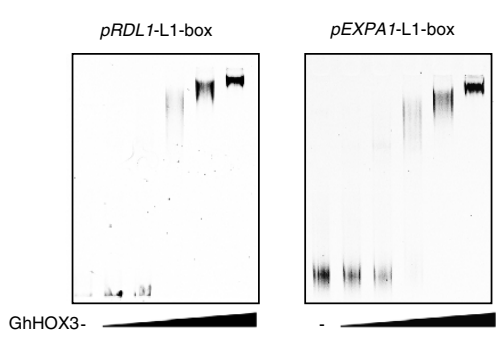

$\mathbf{g}$
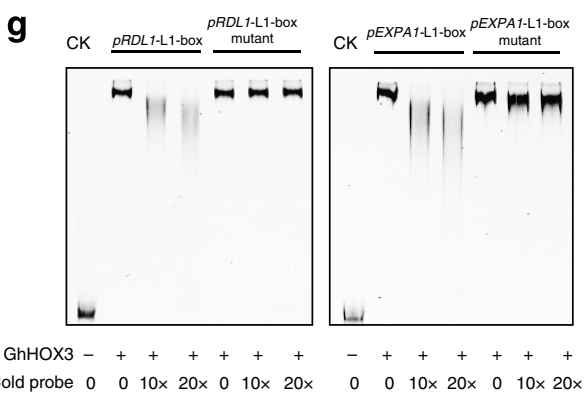

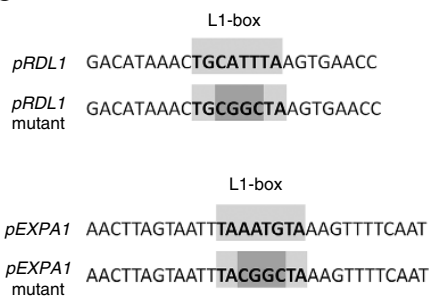

h
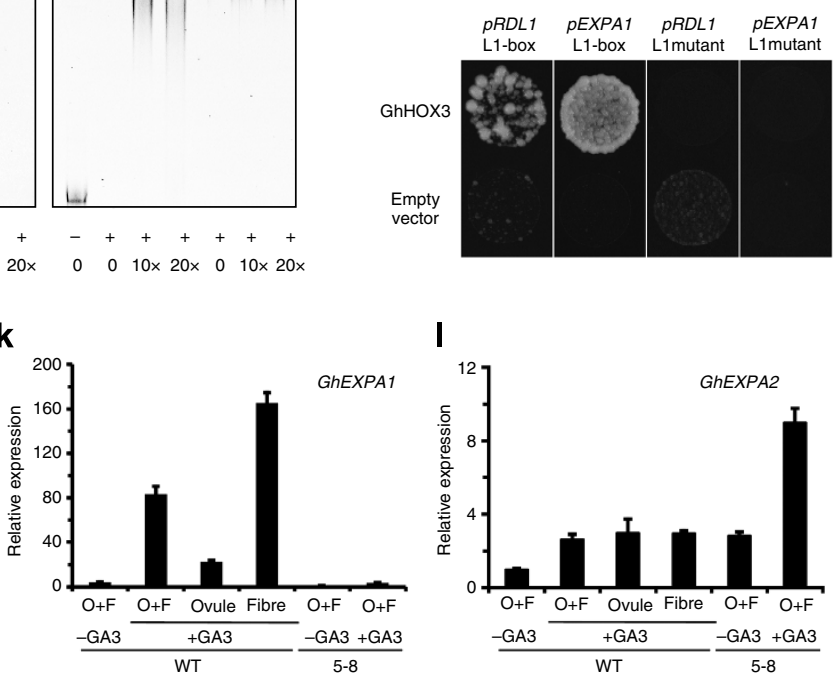

I

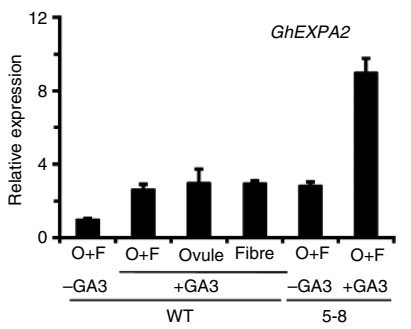

Figure 2 | GhRDL1 and GhEXPA1 are direct targets of GhHOX3. (a-d) Quantitative reverse transcription-PCR analysis of GhRDL1 and GhEXPA1 transcripts in cotton fibre of the WT, co-suppression (5-8) and overexpression (ox1-1) plants. (e-h) GhHOX3 directly binds to GhRDL1 and GhEXPA1 promoters. Data are shown as mean \pm s.e.m. $(n=3)$. (e-g) EMSA of GhHOX3 binding to L1-box from the GhRDL1 and GhEXPA1 promoters. The $6 \times$ fragments of GhRDL1 and GhEXPA1 promoters containing the intact (upper) or the mutated (lower) L1-box (e) were incubated with gradient concentrations of maltose-binding protein (MBP)-GhHOX3 fusion protein (f). Labelled GhRDL1 and GhEXPA1 were incubated with MBP-GhHOX3 to compete with different concentrations of cold probes of intact or mutated L1-box $(\mathbf{g})$. (h) Yeast one-hybrid assay of protein-DNA interaction, the $6 \times$ fragments described in e were used. (i-I) Expression levels of GhHOX3 and two downstream genes in ovule (O) and/or fibre (F), which were taken from GhHOX3-silenced line 5-8 and the WT cotton at 2 DPA and cultured in vitro with addition of the hormone $\mathrm{GA}_{3}(1 \mu \mathrm{M})$ for 6 days. GhEXPA2, expressed at a nearly equal level in ovule and fibre, was analysed as a control of GA treatments. Data are shown as mean \pm s.e.m. $(n=3)$.

this enhancement was synergistic as the combinatorial effect was more than additive (particularly to GhRDL1), and the amounts of agrobacterial cells infiltrated into leaves in each experiment, as well as the amounts of the proteins expressed, were comparable (Supplementary Figs 14,17). In contrast, the promoter activation, either by GhHOX3 alone or by the two HD-ZIP IV proteins in combination, was substantially repressed by GhSLR1 (Fig. 4c). These results were further supported by EMSA assays, in which GhHD1 or GhHOX3 alone, as well as their combination, bound to the L1-box from both GhRDL1 and GhEXPA1 promoters, and such an interaction was indeed disturbed by application of GhSLR1 in the system (Supplementary Fig. 15).

\section{Discussion}

We have shown that the interaction of GhHOX3 with GhHD1 results in a much higher activity of gene activation than either alone, and DELLA (GhSLR1) negatively affects their activity and integration. In plant cells, DELLAs are degraded in response to $\mathrm{GA}^{26}$. Our results uncover a new molecular mechanism underlying the role of GA in promoting cotton fibre elongation (Fig. 5).

PlantTFDB ${ }^{27}$ predicted 15 HD-ZIP IV subfamily transcription factors in the diploid cotton $G$. raimondii genome ${ }^{12}$. At least five of their orthologues are expressed in fibre cells of
G. hirsutum ${ }^{14,15,22}$. Among the four HD-ZIP factors examined here, GhHOX1 is the most similar to, and could functionally substitute for, the trichome regulator AtGL2 in Arabidopsis ${ }^{15}$, whereas GhHOX3 belongs to a distant clade that contains AtHDG11/12 (Supplementary Fig. 16). Overexpression of AtHDG11 in Arabidopsis, tobacco and rice conferred drought tolerance with extensive root architecture change and reduced leaf stomatal density ${ }^{28,29}$. However, our data identify GhHOX3 as a core regulator of fibre elongation, and other HD-ZIP IV proteins, such as GhHD1, could function as accessory factors in the regulatory complex in activating downstream genes of the fibre elongation pathway. Further characterization of the regulatory network centering on GhHOX3 could help to improve cotton fibre quality and yield by increasing fibre length through molecular breeding. Moreover, the direct interaction between DELLA and a specific HD-ZIP IV protein sheds new light on the biochemical mechanism of GA, a widely acknowledged green revolution hormone, in controlling cell elongation, plant height and architecture. Almost simultaneously, a very recent work demonstrated that, during Arabidopsis seed germination, DELLAs affect epidermal cell elongation by sequestering HD-ZIP IV transcription factors from activating downstream gene expression ${ }^{30}$. This result, together with our own, strongly suggests that such mechanism is highly conserved in plants. 


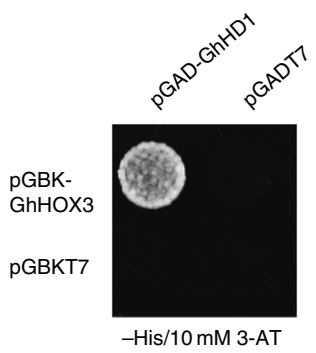

d

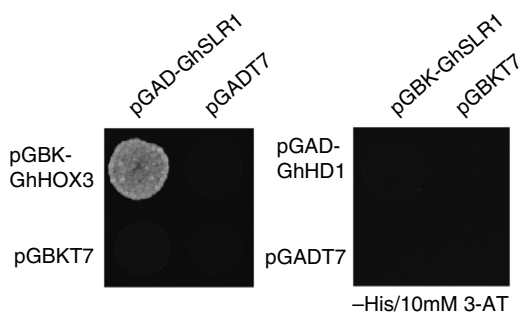

g

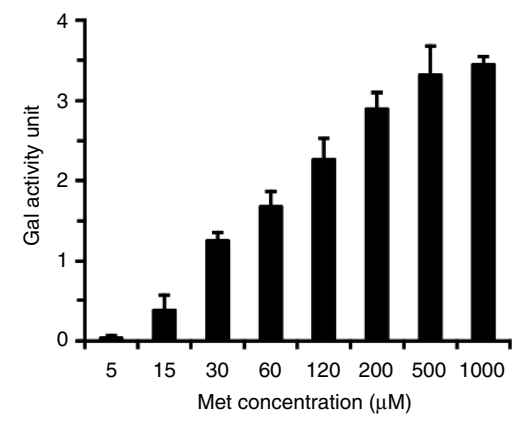

b

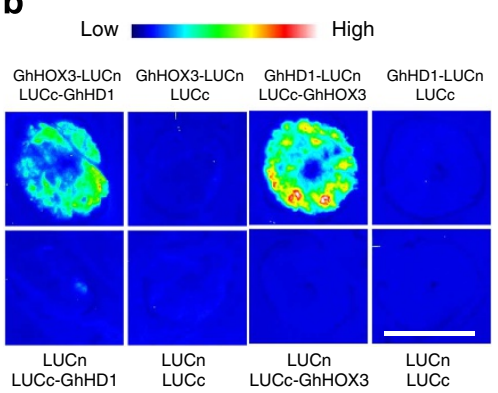

e GhHOX3-LUCn GhHOX3-LUCn GhSLR1-LUCn GhSLR1-LUCn
LUCc-GhSLR1 LUCC LUCc-GhHOX3 LUCc-GhHOX3

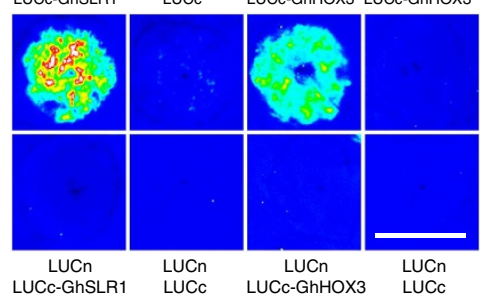

h
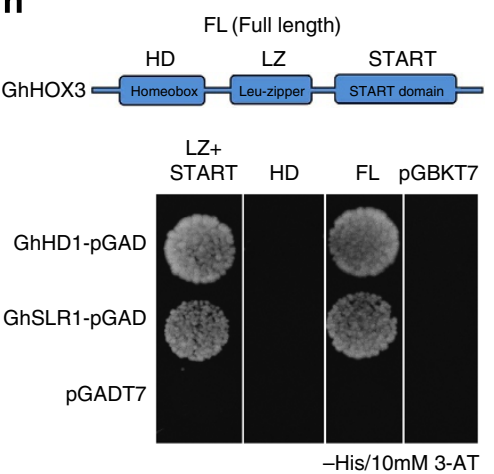

\section{C}
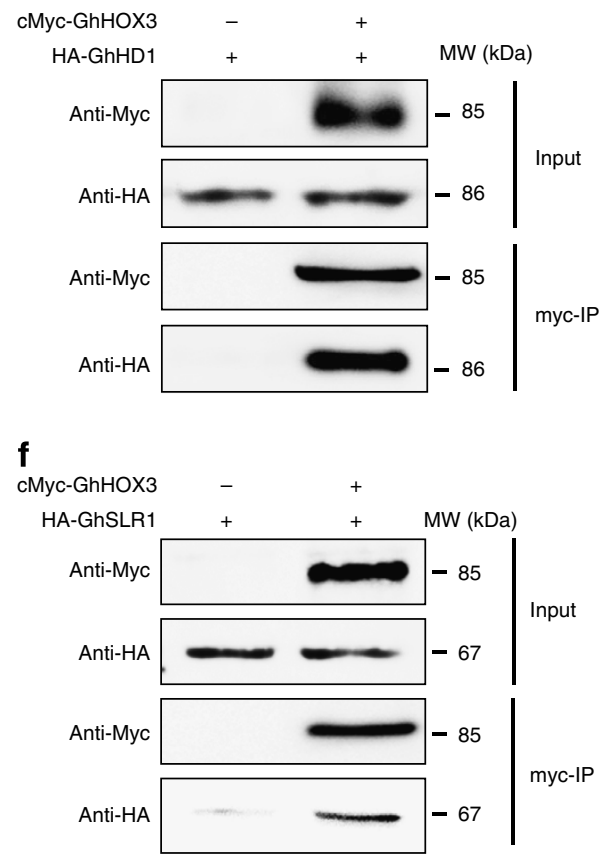

Figure 3 | The DELLA protein GhSLR1 binds to GhHOX3 and interferes with the GhHOX3-GhHD1 interaction. (a) Yeast two-hybrid assay. pGAD-GhHD1 combined with pGBK-GhHOX3 conferred yeast growth on SD/-Leu/-Trp/-His plates supplemented with 10 mM 3-amino-1,2,4-triazole (3-AT).

(b) Bifc assay. GhHOX3 and GhHD1 were interchangeably fused to the carboxyl- and amino-terminal of firefly luciferase (LUC, LUCc and LUCn), transiently co-expressed, and LUCc or LUCn was co-expressed with each other or with each un-fused target protein as the control. Fluorescence signal intensities represent their binding activities. Top bar, heat map's scale of the signal intensity. GhHOX3 interacted with GhHD1. Scale bar, $1 \mathrm{~cm}$ in $\mathbf{b}$. (c) Coimmunoprecipition (CoIP) of transiently co-expressed cMyc-GhHOX3 and HA-GhHD1 in leaves of Nicotiana benthamiana. Soluble protein extracts before (input) and after (IP) immunoprecipitation with anti-cMyc antibody-conjugated beads were detected by immunoblot with anti-HA antibody. (d) Yeast two-hybrid assay. GhHOX3, but not GhHD1, bound to GhSLR1 at $10 \mathrm{mM} 3-\mathrm{AT}$. (e,f) In vivo BiFc (e) and ColP (f) assays. GhHOX3 interacted with GhSLR1. Scale bar, $1 \mathrm{~cm}$ in e. (g) Yeast three-hybrid assay showing the influence of GhSLR1 on GhHOX3-GhHD1 binding represented by $\beta$-galactosidase activity, and the GhSLR1 expression was suppressed by increasing Met concentrations (data are shown as mean \pm s.e.m., $n=3$ ). (h) Domain deletion assay. Top, GhHOX3 contains three conserved domains. Below, yeast two-hybrid detection. GhHOX3 fragment containing both the Leu-zipper (LZ) and the START domains interacted with both GhHD1 and GhSLR1 in yeast, whereas the GhHOX3 homeodomain (HD) did not.

\section{Methods}

Plant materials. Cotton plants (Gossypium hirsutum cv. R15, G. arboreum cv. Qinyangxiaozi, $G$. herbaceum and $G$. raimondii) were grown in a greenhouse at $28 \pm 2{ }^{\circ} \mathrm{C}$ under a 14 -h light photoperiod. Ovules were harvested at $0-18$ DPA at a 3 -day interval. Fibres were collected by scraping the ovule in liquid nitrogen. For genetic analysis, 281 cultivars or lines of G. hirsutum were grown in different areas of China: Akesu (northwestern region), Anyang (Yellow River basin) and Nanjing (Yangtze River basin), respectively, from 2007 to 2009. Nicotiana benthamiana was grown at $22 \pm 2{ }^{\circ} \mathrm{C}$ under a 16 -h light photoperiod.

Genetic analysis. The population comprising of $138 \mathrm{BC}_{1}$ individuals, generated from the cross of $(G$. hirsutum acc. TM- $1 \times G$. barbadense cv. Hai7124) $\times$ TM-01 (ref. 31), was used to map the GhHOX3 gene. According to the different subgenomic sequences between TM-01 and Hai7124, SNP primers for GhHOX3 in corresponding subgenomes were designed to produce the polymorphisms in the two parents. Separation of $G h H O X 3$ in the $\mathrm{BC}_{1}$ mapping population was then detected by polyacrylamide gel electrophoresis (PAGE). Join Map3.0 (ref. 32) was employed to perform the linkage analysis, and the result was integrated with a previously constructed genetic linkage map ${ }^{16}$. MapChart was used to complete the chromosomal localization. Sequences of all primers used in this investigation are listed (Supplementary Table 6). The A-sugenome gene (GhHOX3-A) was used in this investigation unless specifically indicated.

For EcoTILLING assays, young leaves from each cotton variety were freshly harvested for total genomic DNA extraction as described ${ }^{33}$. DNA from all samples was quantified using a spectrophotometer and normalized to a concentration of 20-60 ng $\mathrm{Hl}^{-1}$. The nuclease CEL I was extracted from celery ${ }^{34}$, and enzymatic activity was tested according to the Surveyor Mutation Discovery kit

(Transgenomics) protocol. For nucleotide polymorphism with EcoTILLING, geneand subgenome-specific primers were designed based on the sequence of GhHOX3 by a semi-nested PCR using Ex-Taq polymerase (TaKaRa). The semi-nested PCR for GhHOX3-A used the same forward primer and two different reverse primers. For SNP screens ${ }^{35}$, each of the semi-nested PCR products was mixed with that of TM-1. Heteroduplexes would form in the mixture of each two PCR products if SNPs existed between the variety and TM-1. The heteroduplexes could be digested by CEL I. After cleavage, the heteroduplexes were visualized using PAGE. The cut 

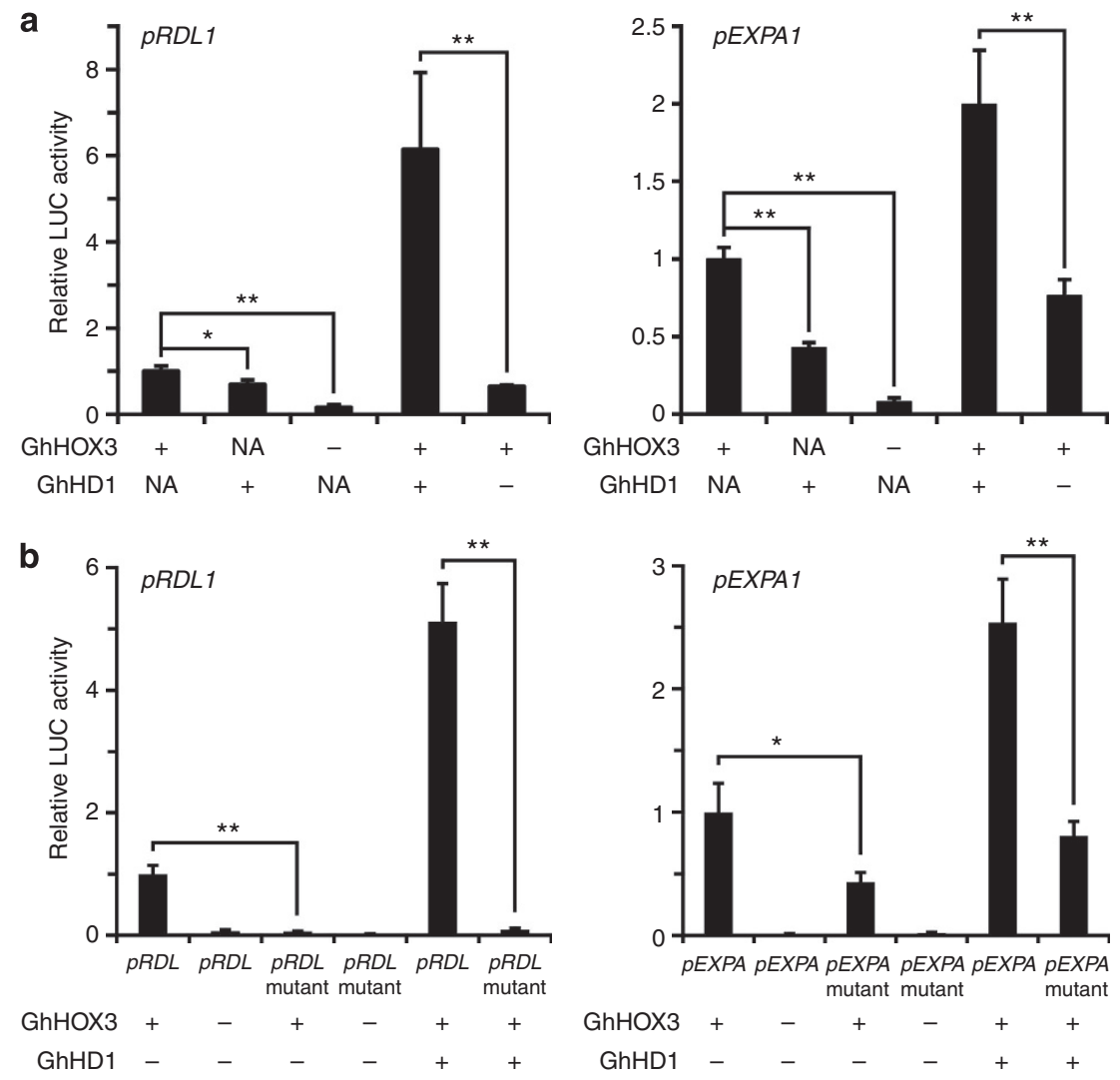

C
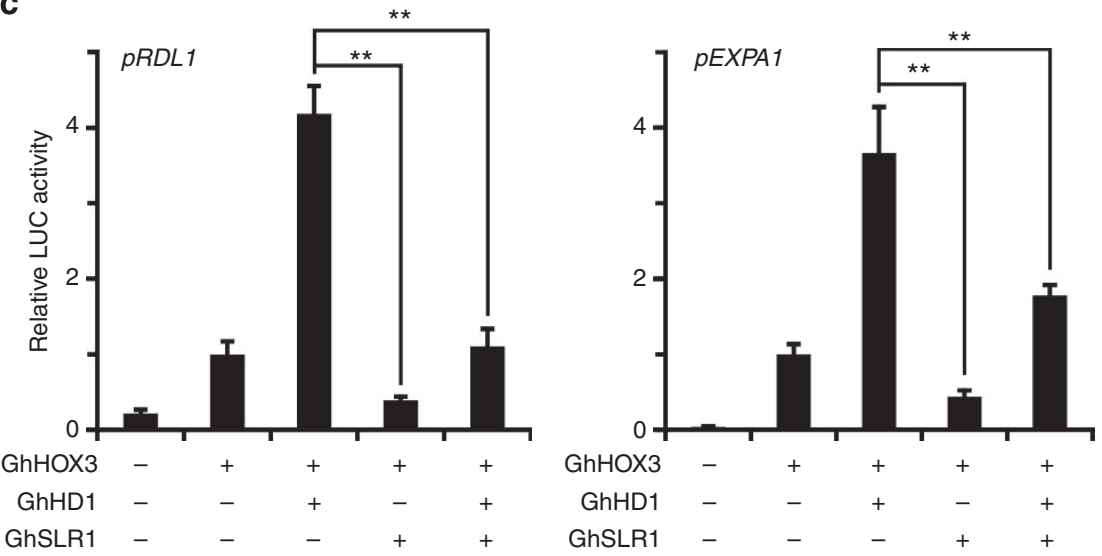

Figure 4 | Transcriptional regulation of target genes by GhHOX3 and the effects of GhHD1 and GhSLR1. GhRDL1 and GhEXPA1 promoters were fused to the LUC reporter, respectively, and the promoter activities were determined by a transient dual-LUC assay in Nicotiana benthamiana. The relative LUC activities were normalized to the reference Renilla (REN) luciferase. The corresponding effector $(+)$, empty vector $(-)$ or neither (NA) were co-filtrated (data are presented as mean \pm s.e.m., $n=3,{ }^{\star} P<0.05$; ${ }^{\star} P<0.01$, Student's $t$-test). (a) Effects of GhHOX3 and GhHD1 on activities of RDL1 and EXPA1 promoters. (b) Effects of L1-box mutation on the activity of RDL1 and EXPA1 promoters. The L1-box was mutated (mutant) as shown (Fig. 2e). (c) Inhibitive effects of GhSLR1 on activation of RDL1 and EXPA1 promoters by GhHOX3 and GhHOX3 + GhHD1.

DNAs were visible as bands and those with faster mobility than the full-length product were considered a polymorphism. Once a polymorphism was identified, the corresponding DNA sample was amplified using gene- and subgenome-specific primers. The resulting PCR fragment was sequenced, and each polymorphic site was sequenced from more than one accession to confirm that only two alleles segregated at any specific site.

Cotton transformation. For the $p 35 S::$ GhHOX3 construct, the open reading frame (ORF) of GhHOX3 was PCR amplified from the G. hirsutum cv. R15 fibre cDNA library using PrimeSTAR HS DNA polymerase (TaKaRa) and cloned into the pCAMBIA2301/35S vector (CAMBIA). For 35S::GhHOX3g, the coding region of GhHOX3-A amplified from genomic DNA was cloned into the $p G W B 5$ vector (Invitrogen). For 35S::dsHOX3, sense and antisense GhHOX3 fragments separated by a 120-bp intron of $A$. thaliana $R T M 1$ gene ${ }^{36,37}$ were cloned into $p B I 121 / 35 S$ (Clontech).

Binary constructs were introduced into Agrobacterium tumefaciens strain LBA4404. The Agrobacterium-mediated cotton transformation was performed using hypocotyl segments of G. hirsutum cv. R15 as explants, as described ${ }^{38}$. After callus induction, proliferation, embryogenic callus induction, embryo differentiation and finally plantlet regeneration, the plantlets were transferred into pots and grown in the greenhouse.

Nucleic acid and expression analysis. Genomic DNA of cotton was isolated with CTAB (cetyltrimethyl ammonium bromide) extraction solution (2\% CTAB, $0.1 \mathrm{M}$ Tris, $20 \mathrm{mM}$ EDTA, $1.4 \mathrm{M} \mathrm{NaCl}, \mathrm{pH}=9.5)($ ref. 39). For Southern blot analysis, 
a

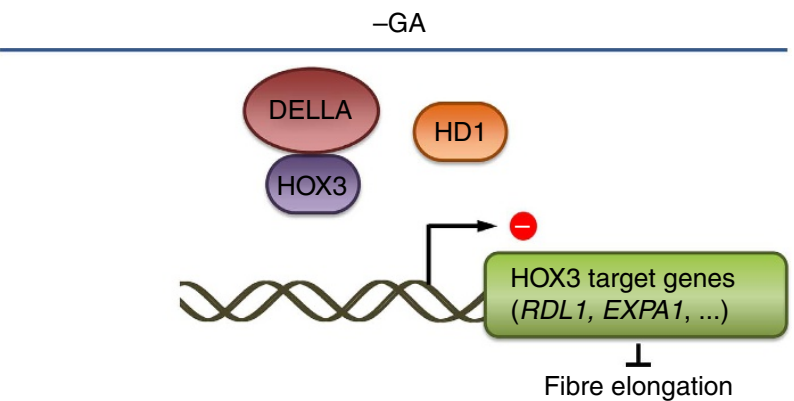

b
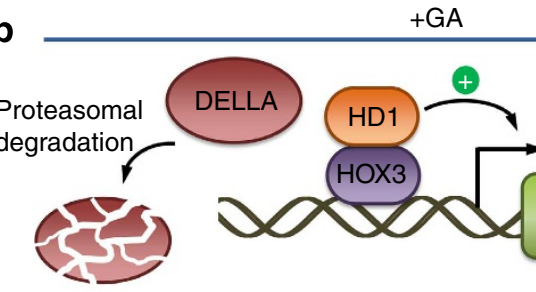

HOX3 target genes
$(R D L 1$, EXPA1, ..)

Fibre elongation

Figure 5 | A model for the regulation of cotton fibre elongation by GhHOX3 and the phytohormone GA. (a) In cotton fibres, DELLA proteins bind to HOX3 to prevent its binding to other HD-ZIP transcription factors, repressing their transcriptional activation to target genes. (b) GAs trigger degradation of DELLAs, releasing HOX3 protein to interact with other HD proteins, such as HD1, allowing the activation of target genes, including RDL1 and EXPA1, to promote cotton fibre elongation.

total genomic DNA samples were digested by restriction enzymes (5U enzyme per $1 \mu \mathrm{g}$ DNA) overnight, separated on $1.0 \%$ agarose gels ( $20 \mu \mathrm{g}$ for each lane) and transferred to Biodyne B membranes (Pall). The fragment of GhHOX3 or the NPTII gene amplified from pCAMBIA2301 (CAMBIA) was used as a probe, which was labelled with ${ }^{32} \mathrm{P}$-dCTP using a Random Primer DNA Labeling kit (TaKaRa). Membranes were hybridized and washed according to a standard protocol.

Cotton RNA was extracted also with the $1 \%$ cetyltrimethyl ammonium bromide (CTAB) solution, precipitated by $2 \mathrm{M} \mathrm{LiCl}^{40}$. For Northern blots, total RNAs $(10 \mu \mathrm{g}$ each lane) were resolved on $1.2 \%$ formaldehyde agarose gels and transferred onto Hybond- ${ }^{+}$nylon membranes (GE Healthcare-Amersham). Hybridization with the ${ }^{32} \mathrm{P}$-labelled probe and membrane washing were performed following standard protocols. Total RNAs of $1 \mu \mathrm{g}$, after treatment with DNase I, were used for cDNA synthesis with oligo(dT) primers and M-MLV (Moloney Murine Leukaemia Virus) Reverse Transcriptase (Invitrogen). Quantitative real-time reverse transcriptionPCR was performed with SYBR-Green PCR Mastermix (TaKaRa), and amplification was real-time monitored on a cycler (Mastercycler RealPlex, Eppendorf). The G. hirsutum histone-3 gene (GhHIS3) was used as an internal reference.

Digital gene expression analysis. Total RNA of 6-DPA fibres from the wild-type (G. hirsutum cv. R15) and GhHOX3 co-suppression line 5-8 was extracted with the the CTAB solution, and the mRNA was separated using oligo-dT magnetic beads, and sheared into short fragments $(\approx 200 \mathrm{bp})$ in the fragmentation buffer. Firststrand cDNA was synthesized by random hexamer primers (mRNA fragments as templates). The double-stranded cDNA was synthesized and purified with a QiaQuick PCR extraction kit (Qiagen), and washed with elution buffer for end repair and single-nucleotide (adenine) addition, followed by the ligation of sequencing adaptors. The fragments were purified by agarose gel electrophoresis and sequenced with a high-throughput sequencer (HiSeq 2000, Illumina) with a read length of $50 \mathrm{bp}$. Three biological replicates were performed separately.

After filtration, cleaned reads were mapped to the G. raimondii genome ${ }^{12}$ using SOAPaligner (SOAP2 (ref. 41), BGI). For annotation, genes mapped were used as query sequences to search against the non-redundant protein database of NCBI, and the Kyoto Encyclopedia of Genes and Genome (KEGG) pathways database. Gene expression levels were normalized and calculated as reads per $\mathrm{kb}$ per million reads values ${ }^{42}$. Significance of differential gene expression was determined (false discovery rate $\leq 0.001$, absolute value of $\log _{2}$ ratio $\left.\geq 1\right)$ by random test $(P<0.05)^{43}$.

Ovule culture. The 2-DPA cotton bolls were harvested and sterilized with $0.1 \%$ $(\mathrm{w} / \mathrm{v}) \mathrm{HgCl}_{2}$ solution for $15 \mathrm{~min}$ and washed three times with sterile distilled water. The sterilized ovules were taken out and placed in liquid BT media, supplemented with different concentrations of auxin (indole-3-acetic acid, IAA, Sigma) and GA
(Gibberellic acid, $\mathrm{GA}_{3}$, Sigma), and cultured in the dark at $30^{\circ} \mathrm{C}$ for 6 days ${ }^{21}$ (Supplementary Figs 10 and 11). Ovules, harvested at 2 DPA, were cultured for 6 days before subsequent analysis.

Yeast assay for protein-protein and protein-DNA interactions. A cDNA library was constructed from mRNAs of the 6-DPA fibres using the CloneMiner II cDNA Library Construction Kit (Invitrogen). The cDNA library was cloned into pDEST22 and GhHOX3 cDNA was inserted into PDEST32. A yeast library screen was performed using GhHOX3-pDEST32 according to the manufacturer's manual (Invitrogen).

Yeast one-hybrid, two-hybrid and three-hybrid analyses were performed using the Matchmaker GAL4 Two-Hybrid System according to the manufacturer's manual (Clontech). Plasmids were transferred into yeast strain AH109 by the LiClPEG method.

For the yeast one-hybrid assay, the $6 \times$ promoter segments of GhRDL1 and GhEXPA1 (intact or mutated L1-box, generated by Generay) were inserted into pHIS2.1 (Clontech), and assayed following the manufacturer's manual. Yeast twohybrid assays were performed with the full-length or truncated ORFs of GhHOX3 inserted into pGBKT7 (Clontech) and GhHD1 or GhSLR1 into pGADT7 (Clontech). Plasmids were co-transferred into yeast, and the interactions were detected on SD/-Leu/-Trp/-His selective plates containing $10 \mathrm{mM}$ 3-AT (3-amino1,2,4,-triazole). Three independent clones for each transformation were tested. Empty vectors of $p G A D T 7$ and $p G B K T 7$ were used as controls. For the yeast threehybrid assay, GhHD1 and GhSLR1 were inserted into pBridge (Clontech), forming a GhHD1-GhSLR1/pBridge construct, and GhHOX3 was placed in $p G A D T 7$. Plasmids were co-transferred into yeast and plated on SD-Leu-Trp selective dropout medium. Colonies were transferred to the appropriate SD/-Leu/-Trp/-His selective dropout liquid medium with different concentrations of methionine (Met). GhSLR1 expression from the pBridge construct was controlled by the pMet25 promoter, and the GhSLR1 level was increased along with the decreasing concentrations of Met. Specific activities of $\beta$-galactosidase were detected according to the manufacturer's manual.

BiFc assay. BiFC assays were performed as previously reported ${ }^{44,45}$. For constructs, ORFs of GhHOX3, GhHD1 and GhSLR1 were PCR amplified and cloned into JW771 and JW772 vectors ${ }^{44}$, respectively. Each ORF was fused to the carboxyl-terminal half (cLUC-GhHOX3/GhHD1/GhSLR1) and the aminoterminal half (GhHOX3/GhHD1/GhSLR1-nLUC) of luciferase (LUC), respectively; cLUC and nLUC were used alone as controls. Agrobacterium cells were resuspended in infiltration buffer $\left(10 \mathrm{mM} \mathrm{MgCl}_{2}, 10 \mathrm{mM}\right.$ MES (2-( $N$-morpholino) ethanesulfonic acid) $\mathrm{pH} 5.7,150 \mu \mathrm{M}$ acetosyringone) at $\mathrm{OD}_{600}=0.8$. 35S::P19-HA and the suspension were co-infiltrated to inhibit gene silencing ${ }^{46}$. After a 3-day culture, a total of $0.8 \mathrm{mM}$ luciferin was infiltrated into the abaxial side of $N$. benthamiana leaves and the LUC activity was monitored. The following pairs of constructs were used for co-infiltration: cLUC-GhHOX3 and GhHD1-nLUC, cLUC-GhHD1 and GhHOX3-nLUC, cLUC-GhHOX3 and GhSLR1-nLUC and cLUC-GhSLR1 and nLUC-GhHOX3, together with their respective controls.

ColP assay. The soluble proteins were extracted using a extraction buffer ( $\mathrm{pH} 7.5$ ) containing $100 \mathrm{mM}$ of Tris- $\mathrm{HCl}, 5 \mathrm{mM}$ EDTA (ethylene diamine tetraacetic acid), $100 \mathrm{mM} \mathrm{NaCl}, 0.2 \%$ Nonidet P-40, $1.0 \%$ Triton-X-100, $1 \mathrm{mM}$ DTT (dithiothreitol), $1 \mathrm{mM}$ PMSF (phenylmethanesulfonyl fluoride), 100 mM MG-132 (Sigma-Aldrich) and protease inhibitor cocktail (Roche). Immunoprecipitation was performed with anti-cMyc-affinity beads (Sigma-Aldrich). Lysates were incubated with the prewashed beads for $1 \mathrm{~h}$ at $4{ }^{\circ} \mathrm{C}$. The beads were then washed three times and solubilized in an appropriate volume of extraction buffer with $5 \times$ SDS loading buffer (Tiangen). GhHOX3-3 $\times$ HA and cMyc-GhHD1/cMyc-GhSLR1 fusion proteins were detected by immunoblot with 1:1,000 diluted anti-HA antibody (Roche) and 1:1,500 diluted anti-Myc antibody (Millipore), respectively.

Protein immunoblot (western blot) analysis. Proteins were separated with $12 \%$ SDS-PAGE and transferred to a polyvinylidene fluoride membrane (GE Amersham). Blots were blocked for $1 \mathrm{~h}$ in PBS, with 5\% skimmed milk powder (Oxoid) and $0.1 \%$ Tween 20 (Sigma-Aldrich), and incubated with corresponding antibody in blocking buffer for $1 \mathrm{~h}$ at room temperature. GhHOX3 monoclonal antibody was raised against the residues 1-200 of GhHOX3 protein (Abmart). After incubation, the blots were washed three times and incubated with 1:10,000 diluted anti-mouse HRP (horseradish peroxidase)-conjugated secondary antibody (Abmart) for $1 \mathrm{~h}$ at room temperature. After washing thrice, the HRP was detected using SuperSignal West Femto Maximum Sensitivity Substrate (Thermo) following the manufacturer's protocol.

EMSA. The ORF of GhHOX3 and GhHD1, in frame, was fused to the maltosebinding protein tag of the expression vector $P M A L-C 2$ (New England Biolabs), and the recombinant proteins were affinity purified following the manufacturer's manual. The ORF of GhSLR1 and the yellow fluorescence protein gene were in frame fused to the glutathione $S$-transferase tag of the expression vector $p G E X-4 T-1$ (GE Healthcare), respectively, and the recombinant proteins were affinity-purified 
using Glutathione Sepharose 4B (GE Healthcare) following the manufacturer's manual. The $6 \times$ promoter segments of GhRDL1 and GhEXPA1, containing the intact or mutated L1-box cis-element, were labelled with Cy5 on both ends. The assay was performed by incubation of the DNA fragment with the purified protein at $25^{\circ} \mathrm{C}$ for $30 \mathrm{~min}$, separated with $5 \%$ native PAGE in $0.5 \times \mathrm{TBE}$ (Tris/Borate/ EDTA) buffer $\left(10 \mathrm{~V} \mathrm{~cm}^{-1}, 4^{\circ} \mathrm{C}\right)$. Fluorescence was observed with an image scanner (FLA-9000, FUJIFILM).

Dual-luciferase (Dual-LUC) assay. The assay was performed as reported ${ }^{47}$. Briefly, The GhRDL1 and GhEXPA1 promoters, with intact or mutated L1-boxes, were inserted into $p$ Green-LUC, respectively, to drive the firefly LUC reporter gene with the Renilla (REN) luciferase controlled by the constitutive 35 s promoter on the same plasmid as a reference to normalize infection efficiency. The constructs were transferred into Agrobacterium tumefaciens (strain GV3101) with the helper plasmid, $p$ Soup-P19, which encodes a repressor of co-suppression. The transformed Agrobacterium cells were mixed with the Agrobacterium strains harbouring the effectors or the empty vector control, in a volume ratio of 1:2.

Transient transformation was conducted by infiltration of the Agrobacterium mixtures into the abaxial side of $N$. benthamiana leaves using a syringe. After culturing for 3 days, the infected area was harvested for total protein extraction. The supernatant of total proteins was used with the Dual-Luciferase Reporter Assay System (Promega) following the manufacture's manual, and the fluorescent values of LUC and REN were detected with a luminometer (BG-1, GEM Biomedical Inc.), successively. The value of LUC was normalized to that of REN. Three biological repeats were measured for each combination.

Microscopic observation. Images were generated with an optical microscope (BX51, Olympus) and fibre length was measured with ImageJ (Wayne Rasband). For subcellular localization, 35S::GFP-GhHOX3-A/D and 35S::GhHOX3-A/D-linker-venus (yellow fluorescence protein) were constructed and transiently expressed in $N$. benthamiana leaves. After 3 days, the leaf tissues were observed under a laser scanning confocal microscope (LSM510, Zeiss). For scanning electron microscope images, cotton ovules (0-2 DPA) were attached with colloidal graphite to a copper stub, frozen under vacuum and visualized with a scanning electron microscope (JSM-6360LV, JEOL)

\section{References}

1. Wilkins, T. A., Rajasekaran, K. \& Anderson, D. M. Cotton biotechnology. CRC Crit. Rev. Plant. Sci. 19, 511-550 (2000).

2. Kim, H. J. \& Triplett, B. A. Cotton fiber growth in planta and in vitro. Models for plant cell elongation and cell wall biogenesis. Plant Physiol. 127, 1361-1366 (2001).

3. Graves, D. A. \& Stewart, J. M. Chronology of the differentiation of cotton (Gossypium Hirsutum L.) Fiber cells. Planta 175, 254-258 (1988).

4. Walford, S. A., Wu, Y. R., Llewellyn, D. J. \& Dennis, E. S. GhMYB25-like: a key factor in early cotton fibre development. Plant J. 65, 785-797 (2011).

5. Machado, A., Wu, Y. R., Yang, Y. M., Llewellyn, D. J. \& Dennis, E. S. The MYB transcription factor GhMYB25 regulates early fibre and trichome development. Plant J. 59, 52-62 (2009).

6. Han, L. B. et al. The dual functions of WLIM1a in cell elongation and secondary wall formation in developing cotton fibers. Plant Cell 25, 4421-4438 (2013).

7. Ruan, Y. L., Llewellyn, D. J. \& Furbank, R. T. Suppression of sucrose synthase gene expression represses cotton fiber cell initiation, elongation, and seed development. Plant Cell 15, 952-964 (2003).

8. Li, X. B., Fan, X. P., Wang, X. L., Cai, L. \& Yang, W. C. The cotton ACTIN1 gene is functionally expressed in fibers and participates in fiber elongation. Plant Cell 17, 859-875 (2005).

9. Xu, B. et al. A cotton BURP domain protein interacts with $\alpha$-expansin and their co-expression promotes plant growth and fruit production. Mol. Plant 6 , 945-958 (2012).

10. Shi, Y. H. et al. Transcriptome profiling, molecular biological, and physiological studies reveal a major role for ethylene in cotton fiber cell elongation. Plant Cell 18, 651-664 (2006).

11. Huang, G.-Q. et al. A fasciclin-like arabinogalactan protein, GhFLA1, is involved in fiber initiation and elongation of cotton. Plant Physiol. 161, 1278-1290 (2013).

12. Paterson, A. H. et al. Repeated polyploidization of Gossypium genomes and the evolution of spinnable cotton fibres. Nature 492, 423-427 (2012).

13. Lin, Q. \& Aoyama, T. Pathways for epidermal cell differentiation via the homeobox gene GLABRA2: update on the roles of the classic regulator. J. Integr. Plant Biol. 54, 729-737 (2012).

14. Wang, S. et al. Control of plant trichome development by a cotton fiber MYB gene. Plant Cell 16, 2323-2334 (2004).

15. Guan, X. Y. et al. The HD-Zip IV gene GaHOX1 from cotton is a functional homologue of the Arabidopsis GLABRA2. Physiol. Plant 134, 174-182 (2008).

16. Guo, W. et al. A microsatellite-based, gene-rich linkage map reveals genome structure, function and evolution in Gossypium. Genetics 176, 527-541 (2007). 17. Taylor, C. B. Comprehending cosuppression. Plant Cell 9, 1245 (1997).
18. Nakamura, M. et al. Characterization of the class IV homeodomain-leucine zipper gene family in Arabidopsis. Plant Physiol. 141, 1363-1375 (2006).

19. Ariel, F. D., Manavella, P. A., Dezar, C. A. \& Chan, R. L. The true story of the HD-Zip family. Trends Plant Sci. 12, 419-426 (2007).

20. Yang, S. S. et al. Accumulation of genome-specific transcripts, transcription factors and phytohormonal regulators during early stages of fiber cell development in allotetraploid cotton. Plant J. 47, 761-775 (2006).

21. Beasley, C. A. \& Ting, I. P. Effects of plant-growth substances on in-vitro fiber development from unfertilized cotton ovules. Am. J. Bot. 61, 188-194 (1974).

22. Walford, S. A., Wu, Y., Llewellyn, D. J. \& Dennis, E. S. Epidermal cell differentiation in cotton mediated by the homeodomain leucine zipper gene, GhHD-1. Plant J. 71, 464-478 (2012).

23. Harberd, N. P., Belfield, E. \& Yasumura, Y. The angiosperm gibberellin-GID1DELLA growth regulatory mechanism: how an 'inhibitor of an inhibitor' enables flexible response to fluctuating environments. Plant Cell 21, 1328-1339 (2009).

24. de Lucas, M. et al. A molecular framework for light and gibberellin control of cell elongation. Nature 451, 480-484 (2008).

25. Aleman, L. et al. Functional analysis of cotton orthologs of GA signal transduction factors GID1 and SLR1. Plant Mol. Biol. 68, 1-16 (2008).

26. Silverstone, A. L. et al. Repressing a repressor gibberellin-induced rapid reduction of the RGA protein in Arabidopsis. Plant Cell 13, 1555-1566 (2001).

27. Jin, J., Zhang, H., Kong, L., Gao, G. \& Luo, J. PlantTFDB 3.0: a portal for the functional and evolutionary study of plant transcription factors. Nucleic Acids Res. 42, D1182-D1187 (2014).

28. $\mathrm{Yu}, \mathrm{H}$. et al. Activated expression of an Arabidopsis HD-START protein confers drought tolerance with improved root system and reduced stomatal density. Plant Cell 20, 1134-1151 (2008).

29. Yu, L. et al. Arabidopsis Enhanced Drought Tolerancel/HOMEODOMAIN GLABROUS11 confers drought tolerance in transgenic rice without yield penalty. Plant Physiol. 162, 1378-1391 (2013).

30. Rombolá-Caldentey, B., Rueda-Romero, P., Iglesias-Fernández, R., Carbonero, P. \& Oñate-Sánchez, L. Arabidopsis DELLA and two HD-ZIP transcription factors regulate GA signaling in the epidermis through the L1 box cis-element. Plant Cell 114, 127647 (2014).

31. Song, X., Wang, K., Guo, W., Zhang, J. \& Zhang, T. A comparison of genetic maps constructed from haploid and $\mathrm{BC} 1$ mapping populations from the same crossing between Gossypium hirsutum L. and Gossypium barbadense L. Genome 48, 378-390 (2005)

32. Ooijen, J. W. \& Voorrips, R. JoinMap: Version 3.0: Software for the Calculation of Genetic Linkage Maps (University and Research Center, 2002).

33. Paterson, A. H., Brubaker, C. L. \& Wendel, J. F. A rapid method for extraction of cotton (Gossypium spp.) genomic DNA suitable for RFLP or PCR analysis. Plant Mol. Biol. Rep. 11, 122-127 (1993).

34. Till, B. J., Zerr, T., Comai, L. \& Henikoff, S. A protocol for TILLING and ecotilling in plants and animals. Nat. Protoc. 1, 2465-2477 (2006).

35. Kadaru, S. B., Yadav, A. S., Fjellstrom, R. G. \& Oard, J. H. Alternative ecotilling protocol for rapid, cost-effective single-nucleotide polymorphism discovery and genotyping in rice (Oryza sativa L.). Plant Mol. Biol. Rep. 24, 3-22 (2006).

36. Johansen, L. K. \& Carrington, J. C. Silencing on the spot. Induction and suppression of RNA silencing in the Agrobacterium-mediated transient expression system. Plant Physiol. 126, 930-938 (2001).

37. Mao, Y.-B. et al. Silencing a cotton bollworm P450 monooxygenase gene by plant-mediated RNAi impairs larval tolerance of gossypol. Nat. Biotechnol. 25, 1307-1313 (2007).

38. Shangguan, X.-X., Xu, B., Yu, Z.-X., Wang, L.-J. \& Chen, X.-Y. Promoter of a cotton fibre MYB gene functional in trichomes of Arabidopsis and glandular trichomes of tobacco. J. Exp. Bot. 59, 3533-3542 (2008).

39. Stewart, Jr C. N. \& Via, L. E. A rapid CTAB DNA isolation technique useful for RAPD fingerprinting and other PCR applications. Biotechniques 14, 748-750 (1993).

40. Yang, C.-Q., Lu, S., Mao, Y.-B., Wang, L.-J. \& Chen, X.-Y. Characterization of two NADPH: cytochrome P450 reductases from cotton (Gossypium hirsutum). Phytochemistry 71, 27-35 (2010).

41. Li, R. et al. SOAP2: an improved ultrafast tool for short read alignment. Bioinformatics 25, 1966-1967 (2009).

42. Mortazavi, A., Williams, B. A., McCue, K., Schaeffer, L. \& Wold, B. Mapping and quantifying mammalian transcriptomes by RNA-Seq. Nat. Methods 5, 621-628 (2008).

43. Benjamini, Y. \& Yekutieli, D. The control of the false discovery rate in multiple testing under dependency. Ann. Stat. 29, 1165-1188 (2001).

44. Gou, J.-Y., Felippes, F. F., Liu, C.-J., Weigel, D. \& Wang, J.-W. Negative regulation of anthocyanin biosynthesis in Arabidopsis by a miR156-targeted SPL transcription factor. Plant Cell 23, 1512-1522 (2011).

45. Chen, H. et al. Firefly luciferase complementation imaging assay for proteinprotein interactions in plants. Plant Physiol. 146, 368-376 (2008). 
46. Papp, I. et al. Evidence for nuclear processing of plant micro RNA and short interfering RNA precursors. Plant Physiol. 132, 1382-1390 (2003).

47. Liu, H. et al. Photoexcited CRY2 interacts with CIB1 to regulate transcription and floral initiation in Arabidopsis. Science 322, 1535-1539 (2008).

\section{Acknowledgements}

We thank J.-W. Wang for discussions; Z.-P. Lin and X.-M. Wu for cotton transformation; X.-Y. Gao and X.-S. Gao for scanning electron microscopy and confocal laser microscopy; S. Wang, H. Hong, W.-R. Cui, W.-T. Li, K. Grupp, J.-X. Li, Z.-X. Yu, X.-Y. Xue, L. Wang and G.-J. Hong for experimental assistance and suggestions; J.-X. Yue for bioinformatic analysis. This work was supported by the State Key Basic Research Program of China (2010CB126004), the Chinese Academy of Sciences (XDB11030300, KSCX2-EW-N-03) and the National Major Project (2014ZX08005-003).

\section{Author contributions}

C.-M.S., T.-Z.Z. and X.-Y.C. conceived and designed the research. C.-M.S., X.-X.S., B.Z., L.-M.C., X.-F.Z., X.-Y.G., C.-Q.Y., H.-Y.Z. and Y.-D.Z. performed the experiments. C.-M.S., C.-Q.Y., L.-J.W., Z.J.C., G.-J.H, J.F.W., W.-Z.G., B.-L.Z. and T.-Z.Z. contributed reagents, materials and/or data analysis. C.-M.S., Z.J.C., T.-Z.Z., J.F.W. and X.-Y.C. wrote the article.

\section{Additional information}

Accession codes: Sequence data for two homoeologous genes of GhHOX3 have been deposited in GenBank/EMBL/DDBJ database under the accession codes KJ595847 (GhHOX3-A) and KJ595848 (GhHOX3-D).

Supplementary Information accompanies this paper at http://www.nature.com/ naturecommunications

Competing financial interests: The authors declare no competing financial interests.

Reprints and permission information is available online at http://npg.nature.com/ reprintsandpermissions/

How to cite this article: Shan, C.-M. et al. Control of cotton fibre elongation by a homeodomain transcription factor GhHOX3. Nat. Commun. 5:5519 doi: 10.1038/ ncomms6519 (2014).

\section{(c) (i)}

This work is licensed under a Creative Commons Attribution 4.0 International License. The images or other third party material in this article are included in the article's Creative Commons license, unless indicated otherwise in the credit line; if the material is not included under the Creative Commons license, users will need to obtain permission from the license holder to reproduce the material. To view a copy of this license, visit http://creativecommons.org/licenses/by/4.0/ 